\title{
Ethnocultural and sex characteristics of patients attending a tertiary care pain clinic in Toronto, Ontario
}

\author{
Angela Mailis-Gagnon MSc MD FRCPC ${ }^{1,2,3}$, Balaji Yegneswaran MBBS ${ }^{1}$, Keith Nicholson PhD 1,3, \\ SF Lakha MSc ${ }^{1}$, Marios Papagapiou MSc ${ }^{1}$, Amanda J Steiman BSc ${ }^{1}$, Danny Ng BSc ${ }^{1}$, Tea Cohodarevic MD FRCPC ${ }^{1}$, \\ Margarita Umana MD¹, Mateusz Zurowski MD
}

\begin{abstract}
A Mailis-Gagnon, B Yegneswaran, K Nicholson, et al. Ethnocultural and sex characteristics of patients attending a tertiary care pain clinic in Toronto, Ontario. Pain Res Manage 2007;12(2):101-106.
\end{abstract}

BACKGROUND: Ethnocultural factors and sex may greatly affect pain perception and expression. Emerging literature is also documenting racial and ethnic differences in pain access and care.

OBJECTIVE: To define the sex and ethnocultural characteristics of patients attending a tertiary care, university-affiliated pain clinic in Toronto, Ontario.

METHODS: Data were collected on 1242 consecutive, new patients seen over a three-year period at the Comprehensive Pain Program (CPP) in downtown Toronto. Data were compared with the Canada 2001 Census.

RESULTS: English-speaking, Canadian-born patients constituted $58.6 \%$ of the CPP population, similar to the 2001 Canadian Census data for the Greater Toronto Area. Certain visible minority groups (Indo-Pakistani and Chinese) were significantly under-represented, while European groups were over-represented. While women outnumbered men, they presented with lower levels of physical pathology in general, particularly in certain ethnic groups. Patients from Europe (representing primarily immigrants who arrived in Canada before 1960), were older, by 10 years to 15 years, than the average CPP population, and had a much higher incidence of physical or medical disorders.

CONCLUSIONS: The implications of the study and the importance of sex and ethnicity in terms of presentation to Canadian pain clinics are discussed. Future well-designed studies are needed to shed light on the role of both patients' and physicians' ethnicity and sex in pain perception and expression, decision-making regarding pain treatments and acceptance of pain treatments.

Key Words: Ethnocultural research; Sex-related factors

E thnocultural variables are well known to play a significant role in pain perception and expression. The relationship of culture or ethnicity and pain has been the focus of research since the 1950s. In both the laboratory and clinical settings, different cultural or ethnic groups seem to react or express pain differently (1-4).

Emerging literature is documenting racial and ethnic differences in pain management for acute, chronic and cancer-related pain, because racial and ethnic minorities seem to be at risk for receiving poor pain assessment and inferior treatments across
Les caractéristiques ethnoculturelles et le sexe des patients fréquentant une clinique de la douleur de soins tertiaires de Toronto, en Ontario

HISTORIQUE : Les facteurs ethnoculturels et le sexe ont peut-être une incidence profonde sur la perception et l'expression de la douleur. Des publications émergentes documentent également des différences raciales et ethniques dans l'accès à la douleur et les soins de la douleur.

OBJECTIF : Définir le sexe et les caractéristiques ethnoculturelles des patients fréquentant une clinique de la douleur de soins tertiaires affiliée à une université de Toronto, en Ontario.

MÉTHODOLOGIE : On a colligé les données auprès de 1242 nouveaux patients consécutifs qui ont été suivis pendant trois ans par le programme intégré de la douleur au centre de Toronto. On a comparé les données avec celles du Recensement du Canada de 2001.

RÉSULTATS : Les patients anglophones nés au Canada représentaient $58,6 \%$ de la population du PID, ce qui est similaire aux données du recensement canadien pour la grande région de Toronto. Certains groupes des minorités visibles (indopakistanais et chinois) étaient très sousreprésentés, tandis que les groupes européens étaient surreprésentés. Les femmes étaient plus nombreuses que les hommes, mais en général, leur pathologie physique était moins grave, notamment dans certains groupes ethniques. Les patients d'Europe (représentant surtout des immigrants arrivés au Canada avant 1960) étaient de dix à 15 ans plus âgés que la population moyenne du PID et avaient une incidence beaucoup plus élevée de troubles physiques ou médicaux.

CONCLUSIONS : On aborde les répercussions de l'étude et l'importance du sexe et de l'ethnie pour ce qui est de la présentation aux cliniques canadiennes de la douleur. De futures études bien conçues s'imposent pour jeter la lumière sur le rôle de l'ethnie et du sexe tant des patients que des médecins dans la perception et l'expression de la douleur, la prise de décision relative aux traitements de la douleur et l'acceptation des traitements de la douleur.

${ }^{1}$ Comprehensive Pain Program; ${ }^{2}$ Krembil Neuroscience Centre, Toronto Western Hospital; ${ }^{3}$ University of Toronto Centre for the Study of Pain, Toronto, Ontario

Correspondence: Dr Angela Mailis-Gagnon, Comprehensive Pain Program, Toronto Western Hospital, 4F811, 399 Bathurst Street, Toronto,

Ontario M5T 2S8. Telephone 416-603-5380, fax 416-603-5725, e-mail angela.mailis@uhn.on.ca 
of most pain conditions is also higher among women than men $(9,10)$. Sex is an especially significant predictor of pain perceptions and coping strategies, because women are more likely to report pain and other symptoms, and to express higher distress about symptoms than men. Women cope more actively with pain by speaking about it, by showing more nonverbal indicators of pain, by seeking spiritual help and by asking more about it (11). Additionally, women are more vulnerable to experimentally induced pain, showing lower thresholds, higher pain discrimination and less tolerance of pain stimuli than men (10). Altogether, the differences between sexes can be attributed to a combination of biological, psychological and sociocultural factors (12-14).

Culture relates to the values, beliefs, norms and practices of a particular group that are learned and shared, and that guide thinking, decisions and actions in a patterned way (15). Symptom- or disease-related beliefs affect patient concerns, anxieties and expectations from the health care process. These beliefs are the interactive product of cultural background, socioeconomic status, educational level and sex. The influence of ethnicity and culture seems to play a substantial role in acute pain expression in children as young as two months (16). It has been shown that in adult patients, ethnicity, culture and religion appear to impact self-reports of illness and pain $(17-19)$. The reporting of physical pain requires some patients to use culturally acceptable idioms through which they can easily communicate their emotional distress to health care providers. Presentation of emotional distress in physical terms has been shown to be associated with stressful events, such as immigration, separation from family, changes in traditional sex roles, financial difficulties and depression (20). It is, therefore, important that health care professionals are able to function under cross-cultural circumstances (cultural competence) (21), because all provider-patient encounters have the potential for cross-cultural misunderstanding, including differing attitudes toward authority, physical contact, communication style, sex, sexuality and family (22).

Therefore, ethnic or racial and sex identifiers may be important in determining who is referred and who is seen by pain clinics, because not only patients' ethnicity and sex, but also physicians' ethnicity and sex may constitute referral biases and alter symptom reports. The present pilot study is the first such data for Canadian pain clinics and provides preliminary insight into the population of a tertiary care, university-affiliated pain clinic in downtown Toronto.

\section{METHODS}

Data were collected for a consecutive series of 1242 new patients referred to the Comprehensive Pain Program (CPP) of the Toronto Western Hospital, a downtown teaching hospital affiliated with the University of Toronto (Toronto, Ontario), over the course of three years (within the 2001 to 2004 period). All demographic information was completed by the patients themselves at the time of consultation. Diagnostic classification data were collected through chart reviews, once the patients were assessed and followed by the pain team physicians. Data collection and analysis for research purposes were approved by the University Hospital Network Research Ethics Board.

Diagnostic categorization used in this clinic was assigned after patient history-taking, clinical examination, review of files and further investigations (if necessary) had been conducted. Physicians working in the CPP routinely use diagnostic categorization of pain disorders along the lines described in the 2000 Diagnostic and Statistical Manual of Mental Disorders, Fourth Edition - Text Revision (23) to facilitate treatment decision-making. Patients, therefore, are classified under one of the following diagnoses: pain disorder associated with a general medical condition, which does not constitute a mental disorder (Group I); pain disorder associated with both psychological factors and a general medical condition (Group II); and pain disorder associated with psychological factors (Group III). In terms of clinical judgment, the CPP physicians consider that a pain disorder associated with psychological factors (Group III) is not a diagnosis of exclusion. It requires a combination of detailed clinical information by history, documentation of positive and negative signs, absence of positive investigations, and behavioural observations including, but not limited to, degree of disability and its concordance with clinical findings and investigations. Further details of the classification methodology are published in the companion paper in this issue of Pain Research 89 Management (pages 93-99).

In terms of ethnic classification, both 'place or country of birth' and 'language spoken at home' were used as ethnic identifiers. This simple classification does not take into account class origin, intermarriages and other ethnic affiliations, years of residency in Canada, and command of English or education level achieved in English. Nevertheless, as an initial attempt to understand the ethnocultural composition of CPP patients, country of birth and language spoken at home were sufficiently strong identifiers to detect trends and guide future research.

The countries of origin were classified based on the 2005 World Population Data Sheet of the Population Reference Bureau (24). For the purposes of the present study, North America included both Canada and USA (the overwhelming majority of such patients were born in Canada; therefore, this group is referred to as the Canadian group); southern Europe cumulatively included the Mediterranean countries (Greece, Italy, Portugal, Spain, etc); northern Europe (Denmark, Finland, Norway, Sweden and the United Kingdom) nearly exclusively included patients born in the United Kingdom; the Caribbean primarily included patients from Jamaica, Trinidad and Tobago, and the Dominican Republic; eastern Europe included patients born primarily in Poland, Romania, Russia, Slovakia and Ukraine; south-central or south Asia included patients born primarily in India, Iran and Pakistan (the vast majority Indo-Pakistani patients), and east Asia included patients born in China and Hong Kong. For certain comparisons, the three European groups identified in the CPP population (northern, southern and eastern European patients) were lumped together to increase the sample size.

The Greater Toronto Area (GTA) is a region of $7000 \mathrm{~km}^{2}$ that presently includes 25 municipalities, four regions and 5.6 million people ( $41.8 \%$ of the total population of Ontario). Whenever possible, the study data were compared with the GTA statistics of the 2001 Census of Canada (unless otherwise specified), even if some CPP patients were referred from outside the GTA. For example, the proportion of each ethnic group as defined in the present study was compared with the total number of GTA residents born in the specific country of origin divided by the total number of GTA residents for the 2001 Census (4,893,290 individuals). Statistical analyses were conducted whenever possible to assess whether relationships were statistically significant using the appropriate statistics $\left(\mathrm{eg}, t, \mathrm{~F}, \chi^{2}\right)$; however, this is a retrospective study using a fairly large sample but no specific hypothesis testing. As such, these statistics (when mentioned in the text) provide only some indication, with at least a $\mathrm{P}<0.05$ level of statistical significance, of results that may not simply be due to chance distribution of the data. 


\begin{tabular}{|c|c|c|c|c|}
\hline Group & Birth/ethnicity & $\begin{array}{c}\text { Number of } \\
\text { patients } \\
(n=1242)\end{array}$ & $\begin{array}{c}\text { Per cent } \\
\text { of CPP } \\
\text { population }\end{array}$ & $\begin{array}{c}\text { Per cent } \\
\text { of } 2001 \text { GTA } \\
\text { population }\end{array}$ \\
\hline 1 & North American & 729 & 58.60 & 57.70 \\
\hline 2 & Southern European & 121 & $9.74(86)^{\star}$ & 5.63 \\
\hline 3 & Northern European & 51 & $4.11^{\dagger}$ & 3.23 \\
\hline 4 & Caribbean & 49 & $3.95(90)^{*}$ & 4.25 \\
\hline 5 & Eastern European & 43 & $3.46(81)^{*}$ & 3.62 \\
\hline 6 & South-central Asian & 48 & $3.86(79)^{*}$ & 6.69 \\
\hline \multirow[t]{2}{*}{ Total } & & 1041 & 83.62 & \\
\hline & East Asian $\ddagger$ & 20 & 1.60 & 6.01 \\
\hline
\end{tabular}

*Numbers in brackets indicate the percentage of foreign-born immigrants who spoke the language of country of origin at home. 'The northern Europeans spoke English because they almost all came from the United Kingdom. ¥This small group is specifically mentioned, because it was seriously under-represented. GTA (residing in the) Greater Toronto Area

\section{TABLE 2}

Female to male ratio

\begin{tabular}{ll}
\hline Birth/ethnicity & Ratio \\
\hline Canadian & $1.30: 1$ \\
European* & $1.65: 1$ \\
Caribbean & $1.93: 1$ \\
South-central Asian & $0.80: 1$ \\
\hline
\end{tabular}

${ }^{*}$ All three European groups were analyzed together to increase the sample size

\section{RESULTS}

Of 1242 patients seen over a three-year period, 727 patients were born in Canada. Of the 727 patients, 703 spoke English and 14 spoke French as their first language at home. Two patients were born in the USA and were lumped together into the large 'Canadian' group. The rest of the patients were born outside of Canada or the USA and are termed collectively in the present study as 'foreign born.'

Group classification based on country of birth

Greater detail on the six largest groups with adequate numbers for analysis, which constituted $83.62 \%$ of the CPP population, is reported in Table 1. Based on the place of birth, the Canadian group comprised $58.56 \%$ of the CPP population (similar to the GTA data of the 2001 Census of Canada), followed by the southern European group (9.74\%), the northern European group $(4.11 \%)$, the Caribbean group $(3.95 \%)$, the south-central Asian group (3.86\%) and the eastern European group (3.46\%). Other ethnic groups were seen in much smaller proportions, including the east Asian (primarily Chinese) group, which constituted only $1.6 \%$ of our patient population. Altogether, the foreign-born group, as defined above, accounted for $41.4 \%$ of the CCP population. Table 1 shows the data in detail and compares it with GTA data. When the data with regard to language spoken at home were analyzed, the majority of foreign-born patients ( $79 \%$ to $90 \%)$ spoke the language of the country of origin at home (data shown in Table 1).

\section{Sex differences}

Sex ratios between male and female referrals are seen in Table 2. Women, in general, outnumbered men in almost all
TABLE 3

Age at presentation

\begin{tabular}{|c|c|c|}
\hline Birth/ethnicity & Mean age, years & Comment \\
\hline Canadian & 45.7 & Female age equal to male age \\
\hline Southern European & 56.1 & Female age equal to male age \\
\hline Eastern European & 63.4 & $\begin{array}{l}\text { Men were } 5.5 \text { years older than } \\
\text { women }\end{array}$ \\
\hline Northern European & 57.0 & Female age equal to male age \\
\hline Caribbean & 46.3 & $\begin{array}{l}\text { Men were } 10 \text { years older than } \\
\text { women }\end{array}$ \\
\hline South-central Asian & 48.5 & Female age equal male age \\
\hline
\end{tabular}

TABLE 4

Education (based on some or highest level of education achieved)

\begin{tabular}{lcc}
\hline Birth/ethnicity & High school, \% & College/university, \% \\
\hline Canadian & 41 & 44 \\
Southern European & 29 & 11 \\
Eastern European & 14 & 43 \\
Northern European & 37 & 47 \\
Caribbean & 48 & 32 \\
South-central Asian & 34 & 42 \\
\hline
\end{tabular}

groups, with the female to male ratio ranging between $1.3: 1$ to 1.93:1, except in the south-central Asian (Indo-Pakistani) group, in which men slightly outnumbered women.

Age at consultation

At the time of presentation to the CPP, the Canadian, Caribbean and south-central Asian patients had a mean age ranging from 45.7 years to 48.5 years (Table 3 ). The mean age was similar between men and women in all groups except the Caribbean group, in which men were older by 10 years than females; quite noticeably, however, the mean age of the three European groups ( 57.8 years) was significantly older than the mean age of the other groups (45.9 years; $t=10.78$, $\mathrm{P}<0.001$ ) when analyzed, cumulatively, for both sexes.

Education obtained

Canadian and most foreign-born patients were quite well educated (Table 4) (at par with the Toronto 2001 Census data, which indicated that $49.6 \%$ of the working age population had attained at least some college or university education). The exception was the group of patients coming from southern Europe, of whom only $11 \%$ had such education.

Diagnostic classification

Statistical analysis confirmed that there was a significant difference in primary pain diagnosis across ethnic groups (Pearson's $\chi^{2}=24.4, \mathrm{P}<0.01$ ). In terms of underlying pathology (Table 5), $25.9 \%$ of Canadian-born patients were classified in Group I with easily detectable pathology. Very high levels of physical pathology were seen in the eastern European and southern European groups (47.5\% and 29.8\%, respectively), while the south-central Asian group had the lowest levels of such pathology $(18.4 \%)$. Patients in Group II, with mixed medical pathology and nonmedical factors contributing to disability, accounted for one-half of all patients seen (ranging from $40 \%$ to $50.9 \%$ for each ethnic group). Patients in 
Group III, with significant pain disability in the absence of relevant detectable pathology, again varied substantially in numbers among ethnic groups, and ranged from a low of $11.4 \%$ (for the eastern European group) to a high of $31.6 \%$ for the southcentral Asian group. Furthermore, in this diagnostic class, women clearly outnumbered men in most of the ethnic groups. A special note should be made with regard to the large numbers of south-central Asian (Indo-Pakistani) and Caribbean women who were classified into Group III (47\% and 37.9\%, respectively).

Interestingly, failed back surgery syndrome (FBSS) (male to female ratio 3:2) was much more prevalent in Canadian- than foreign-born men $(2: 1)$, while there was no such difference in women.

\section{DISCUSSION}

The present study of 1242 consecutive, new chronic pain patients attending a university-affiliated chronic pain clinic in downtown Toronto demonstrated that: Canadian-born patients constituted $58.6 \%$ of the CPP population (data similar to the GTA data from the 2001 Census); certain foreignborn groups were significantly under-represented, while others were over-represented; the CPP population was well educated, similar to the Toronto average; while women significantly outnumbered men, they presented with lower levels of physical pathology, based on our system of diagnostic classification, particularly in two visible minority groups; and while the average age of most patients was approximately 45.7 years to 48.5 years at the CPP presentation, patients from Europe were older by 10 years to 15 years and had a much higher incidence of physical or medical disorders.

\section{Understanding the multicultural roots of Canada}

To interpret the results of the present study, one needs to comprehend the multicultural roots of Canadian society. The 2001 Census of Canada (25) showed that the national average of foreign-born people was $18 \%$; however, this varied among provinces and urban or rural areas. In Ontario, the most populated Canadian province in 2001, 27\% of the total population was born outside of the country, with a variable degree of visible minorities across regions. Visible minorities were defined as "persons (other than Aboriginals) who are nonCaucasian in race or nonwhite in colour" (25). In 2001, Ontario was home to $38 \%$ of the total Canadian population and had the largest share of all incoming immigrants for that year (56\% of all ethnic groups), because immigrants tend to concentrate in the biggest cities in the country. For Toronto, the largest city in Ontario and Canada, the percentage of foreignborn people $(44 \%)$ is actually higher than that in Miami, Sydney, Los Angeles or New York, and more than twice as high as Canada's national average. A very important consideration is the fact that the types of immigrants making Toronto their home has changed over the years. Before 1961, 92\% of the immigrants came from Europe and only 3\% from Asia; however, in the 1990s, the trend completely reversed, as 79\% of the immigrants who arrived in Toronto during the 1990s were visible minorities (primarily Asian), while Europeans accounted for only $17 \%$ of the immigrants. In 2001, visible minorities specifically comprised $36.8 \%$ of Toronto's total population. The largest visible minority group is the south Asian or southcentral Asian group (eg, Indian, Iranian and Pakistani), followed by the east Asian group (Chinese) and the black
TABLE 5

Disorder classification per group (based on country of birth and population percentage)

\begin{tabular}{|c|c|c|c|c|c|c|c|c|c|}
\hline \multirow[b]{2}{*}{ Birth/ethnicity } & \multicolumn{3}{|c|}{ Group I } & \multicolumn{3}{|c|}{ Group II } & \multicolumn{3}{|c|}{ Group III } \\
\hline & $F$ & $\mathbf{M}$ & $F+M$ & $\mathbf{F}$ & $\mathbf{M}$ & प & $\mathbf{F}$ & $\mathbf{M}$ & $F+M$ \\
\hline dint & 23.3 & 29.4 & 25.9 & 51.5 & 50.2 & 50.9 & 22.3 & 0.0 & 19. \\
\hline outhern European & 24.2 & 38.0 & 29.8 & 48.4 & 45.2 & 47.1 & 24.2 & 14.3 & 20.2 \\
\hline & 38.0 & 57.0 & & 47.6 & 28.6 & & 9.5 & 14.3 & 11.4 \\
\hline lorthern European & 23.5 & 13.3 & 20.4 & 50.0 & 40.0 & 46.9 & 23.5 & 40.0 & 28.6 \\
\hline All European & 36.5 & 36.6 & 30.3 & 48.7 & 40.8 & 45.7 & 21.4 & 19.7 & 20.7 \\
\hline Caribb & 13.8 & 33.3 & 20.5 & 44.8 & 53.3 & 47.7 & 37.9 & 13.3 & 29.6 \\
\hline outh-central Asian & 11.8 & 23.8 & 18.4 & 41.2 & 57.0 & 50.0 & 47.0 & 19.0 & 31.6 \\
\hline
\end{tabular}

Group I Pain disorder associated primarily with biomedical factors; Group II Pain disorder associated with a general medical condition and psychological factors; Group III Pain disorder associated with psychological factors. F Female; $M$ Male

community, comprising $10.2 \%, 9 \%$ and $7 \%$ of the total population of Toronto, respectively. In terms of educational profile, the 2001 Census shows that recent male and female immigrants (after 1990) were highly educated. Cumulatively, in Toronto, the 2001 Census indicates that $17.5 \%$ of the population aged 25 years to 64 years had some college education and $32.1 \%$ had some university education (the data include both Canadian-born and foreign-born people). While Ontario is the highest educated province in Canada, Toronto's population percentage with some university education is even higher than that of Ontario.

\section{Differences in age of presentation}

While the peak age of pain patients presenting to the CPP (pages 93-99 in this issue of Pain Research 8 Managment) is in the 35- to 49-year age group, the European subgroups, individually and collectively, were much older than all the other groups, including the Canadian-born CPP population. The high levels of biomedical pathology, particularly in the eastern and southern Europeans, may be explained by the significantly older age of these patients (in accordance with generally published data indicating that painful disorders advance with age) and by the fact that these early immigrants were often working in physically demanding jobs, potentially leading to more severe degenerative changes. However, the delayed presentation of Europeans to our pain clinic, despite the presence of significant biomedical pathology, in our view, may depend more on psychosocial factors, such as patient attitudes ('grin and bear it', accepting pain as a 'natural consequence of age' or lower 'entitlement' beliefs) or referring physicians' preferences.

\section{Differential attendance of ethnic groups}

The attendance of certain ethnic groups to the CPP seemed to be uneven compared with the GTA data of the 2001 Census. Specifically, the east Asian group (primarily Chinese) was the most seriously under-represented in our patient population (1.6\% of the CPP population), despite that this group accounted for $9 \%$ of the population in Toronto and $6.01 \%$ of the GTA population, and that our clinic was located in the middle of the largest Chinese community in Toronto. Data in the current study are similar to unpublished data from our clinic 15 years previously, when we noticed that this group, specifically, was sparse in attendance. This serious under-representation may be due to a 
number of factors, such as Chinese patients' preference for referral to traditional Chinese clinics rather than Western health care services (based on personal communication with Chinese health care providers and unpublished data); referral bias based on referring physicians' preferences; and potential differences in the prevalence of chronic pain in this ethnic group. The latter point is supported by the Women's Health Surveillance Report from Statistics Canada (8), which reported that Chinese patients older than 65 years had the lowest chronic pain prevalence than any other ethnic group in the Canadian population. In summary, genetic and psychosocial factors (ie, attitudes and beliefs, personal preferences, etc) may account for the disproportionate presentation of this ethnic group to our tertiary care pain clinic.

The south-central Asian (Indo-Pakistani) group seemed to be under-represented as well, and constituted $3.86 \%$ of the CPP population, versus $10.2 \%$ in Toronto and $6.69 \%$ in the GTA. While one can argue that attendance, underlying pathology and age of presentation for all groups may be affected by a combination of biomedical, psychological and ethnocultural factors, there are certain differences in this particular group (with regard to underlying pathology for both sexes and women in particular) that may point more to sociocultural variables specifically affecting their pain complaints, as is discussed further below.

Compared with the south-central and east Asian groups, the southern European group seemed to be over-represented at 9.74\% of the CPP population, versus $5.76 \%$ of the GTA population. In our view, the high level of physical pathology seems to be the predominant driver for the attendance of the southern and eastern European groups to the CPP, while older age at presentation may be accounted for by psychosocial factors, as discussed earlier.

\section{Educational attainment}

The general level of education of the CPP population is generally consistent with the 2001 Census of Canada report (25) that the most recent waves of immigrants are highly educated. While the numbers in our study are very small for statistical analysis, the lower education of the southern European group, presumed to have originated from the 1960s wave of immigration, seems to be in accordance with the types of physical labour jobs many of these patients had when they immigrated (food industry, construction, etc). However, it should be noted that our data on foreign-born patients do not allow us to ascertain whether their highest education was achieved in the country of origin or in Canada and their mastery of the English language; therefore, our educational data may not be directly comparable between Canadian- and foreign-born individuals. The observation that the vast majority of foreignborn patients spoke the language of country of birth at home does not negate the fact that many immigrants were also proficient in English. Nevertheless, properly designed studies are needed to explore language issues as part of acculturation.

\section{Sex differences}

In general, the predominance of women in our CPP population is on par with previous (unpublished) data in this clinic in past years, as well as with several epidemiological studies. Women consistently outnumbered men in nearly all ethnic groups in our study and presented with less physical pathology, reflecting a higher number of women classified into Group III.
Biomedical, psychological and sociocultural variables may account for these differences, as was noted in the introduction. An interesting observation is that $47 \%$ of the south-central Asian women and $38 \%$ of the Caribbean women were classified into Group III, in which no physical pathology could be ascertained for their pain complaints and psychosocial factors contributing to presentation were apparent. Our findings that the perception and expression of pain may be enhanced in the south-central Asian group may be supported by the following observations. A study of thermal pain responses in white, British and south (central) Asian, healthy men showed no differences between the two groups in cold and warm sensory perception thresholds (ie, no physiological differences). However, the south Asians showed lower pain thresholds to heat and greater pain sensitivity. It was concluded that ethnicity plays an important role, even if the investigators were not exactly sure what determinants of ethnicity (behavioural, genetic, etc) were involved (4). In the Women's Health Surveillance Report from Statistics Canada, which surveyed approximately 100,000 households (8), the proportion of south-central Asians who reported chronic pain was much greater than any other ethnic group in the Canadian population older than 65 years (with $38.2 \%$ of the men and $55.7 \%$ of the women reporting chronic pain). It may be that culturally based expression of emotional distress, in physical terms, may contribute to this enhanced pain expression (20), particularly taking into account stress of immigration (because south-central Asians constitute the newer wave of immigrants to Ontario).

Despite the small numbers of FBSS patients, it was interesting to note the higher prevalence of FBSS in Canadian-born versus foreign-born men, because the data support our clinical impression that foreign-born patients 'are not too keen to have their back operated on.' While other factors (eg, not proposing surgery as a therapeutic option to certain groups) $(5,6)$ may account for the observed differences, it has also been shown that some ethnic groups are more prone to decline certain surgical interventions when offered (26). Nevertheless, it would be interesting to determine the ethnic constitution of FBSS patients in other pain clinics, where the syndrome is stated to account for almost one-quarter of all patients seen (Dr Dwight Moulin, personal communication).

\section{Difficulties associated with ethnocultural research}

Clearly, ethnocultural research may face several difficulties. For example, when Americans are grouped into blacks, Hispanics and old Americans (white Anglo-Saxons whose families have been in the United States for several generations) (27), one cannot help but notice that millions of black Americans' ancestors were brought to America long before many of the people currently fitting the category of 'old Americans'. Furthermore, the definition of 'blacks' does not allow for comprehension of the massive social, cultural and economic differences between descendents of people brought to America two to three centuries ago and the millions of recent immigrants from the Caribbean and Africa (28), nor to what extent these populations have acculturated to the dominant social ethic. A further concern in a multicultural society is the manner in which one takes into account the massive proportion of the population that is of mixed background through intermarriage.

Within any ethnic group, class and sex are as important as ethnicity itself $(29,30)$. The stereotype of older immigrants was 
that they were coming to the western world from impoverished countries to 'start from the bottom'. However, more recent waves of immigrants from many ethnic groups come from very affluent backgrounds, and may have very different social and cultural views, including views toward health issues (31), than those from different classes from the same place of origin. It may, in fact, be specifically the lower socioeconomic status that places racial minorities at increased risk for inappropriate or lesser treatment $(32,33)$. Therefore, well designed studies must take into account all these factors (degree of acculturation, class and socioeconomic variables, sex, etc) to provide meaningful results.

The need for recognition of racial and ethnic identifiers is stressed by Williams (34) to document pain disparities in clinical settings based on retrospective data; to plan and implement prospective studies to detect disparities; to develop and evaluate pain assessment tools that reflect cultural, ethnic and linguistic diversities; to understand the role of both patients' and physicians' ethnicity in pain management decision-making; to examine racial and ethnic differences in pain perception, beliefs, attitudes and behaviours that may underlie differences in pain experiences and clinical pain conditions; to develop culturally sensitive models for assessing and treating pain (35), and methods to disseminate such information; and to document progress toward eliminating disparities in pain management, and evaluate pain management outcomes.

\section{Limitations}

We should stress that our study has several limitations, including the following: the data were retrospective and some were missing; detailed indicators of the degree of acculturation (intermarriages, length of stay in Canada, multiple ancestry, command of English language, etc) were not collected; some subgroups were too small for statistical analysis; the comparison between our data and the GTA data of the 2001 Census may provide a source of limited error, because our 'catchment area' (the area from which patients are referred to us) is quite large, extending outside of the GTA. However, given the fact that immigrants are largely concentrated in the big cities, we felt that comparison of our data to the GTA data was the best available way to understand ethnocultural prevalence of chronic pain in our clinic; furthermore, the comparison of the 2001 Census of Canada data to those of our study may also account for limited error, because our data were collected later than 2001 (2001 to 2004 period). However, the 2001 Census statistics were the only ones available for comparison, and the results of the present study are not necessarily generalizable to other Canadian pain clinic populations given the fact that other pain clinics may be very different in terms of staff, diagnostic or management approaches and therapeutic modalities, as well as the types of patients they attract and treat. Furthermore, referral biases based on multiple factors (location, availability, waiting list, referring physicians' preferences, ethnocultural factors relating to both referring physicians and patients, specialization and expertise, etc) do influence the types and numbers of patients attending a given pain clinic.

Nevertheless, this is the first published data for Canadian pain clinics, pointing to the need for further well-designed studies to assist in the development of a biocultural model, which may be helpful in conceptualizing the complex interaction of biological, cultural and psychosocial factors in pain perception (36-38). The improvement of pain care for all ethnic or racial groups can be achieved in mixed societies, if ethnoculturally specific and well-designed studies are performed to assist in the development of cultural competence (21) and to guide potential interventions (39).

\section{REFERENCES}

1. Hobara M. Beliefs about appropriate pain behavior: Cross-cultural and sex differences between Japanese and Euro-Americans. Eur J Pain 2005;9:389-93.

2. Korol CT, Craig KD. Pain from the perspectives of health psychology and culture. In: Kazarian SS, Evans DR, eds. Handbook of Cultural Health Psychology. San Diego: Academic Press, 2001:241-65.

3. Rollman GB. Ethnocultural variations in the experience of pain. In: Hadjistavropoulos T, Craig KD, eds. Pain: Psychological Perspectives. Mahwah, New Jersey: Lawrence Erlbaum Associates, 2004:155-78.

4. Watson PJ, Latif RK, Rowbotham DJ. Ethnic differences in thermal pain responses: A comparison of South Asian and White British healthy males. Pain 2005;118:194-200.

5. Smedley BD, Stith AY, Nelson AR. Unequal Treatment: Confronting Racial and Ethnic Disparities in Health Care. Washington, DC: National Academy Press, 2003.

6. Green CR, Anderson KO, Baker TA, et al. The unequal burden of pain: Confronting racial and ethnic disparities in pain. Pain Med 2003;4:277-94. (Erratum in 2005;6:99).

7. Robinson ME, Riley III JL, Myers CD. Psychosocial contributions to sex-related differences in pain responses. In: Fillingim RB, ed. Sex, Gender, and Pain: From the Bench Top to the Clinic. Amsterdam: International Association for the Study of Pain, 2000:41-68.

8. Meana M, Cho R, DesMeules M. Chronic Pain: The Extra Burden on Canadian Women. BMC Womens Health 2004;4(Suppl 1):S17.

9. Unruh AM. Gender variations in clinical pain experience. Pain 1996;65:123-67.

10. Berkley KJ. Sex differences in pain. Behav Brain Sci 1997;20:371-80.

11. Affleck G, Tennen H, Keefe FJ, et al. Everyday life with osteoarthritis or rheumatoid arthritis: Independent effects of disease and gender on daily pain, mood, and coping. Pain 1999;83:601-9.

12. Jablonska B, Soares JJ, Sundin O. Pain among women: Associations with socio-economic and work conditions. Eur J Pain 2006; 10:435-47

13. Wiesenfeld-Hallin Z. Sex differences in pain perception. Gend Med 2005;2:137-45.

14. Keogh E. Sex and gender differences in pain: A selective review of biological and psychosocial factors. J Men Health Gend, 2006;3:243-36.

15. Leininger M. Qualitative research methods in nursing. Orlando: Grune \& Stratton, 1985.

16. Rosmus C, Johnston CC, Chan-Yip A, Yang F. Pain response in Chinese and non-Chinese Canadian infants: Is there a difference? Soc Sci Med 2000;51:175-84

17. Juarez G, Ferrell B, Borneman T. Cultural considerations in education for cancer pain management. J Cancer Educ 1999; $14: 168-73$.

18. White SF, Asher MA, Lai SM, Burton DC. Patients' perceptions of overall function, pain, and appearance after primary posterior instrumentation and fusion for idiopathic scoliosis. Spine 1999;24:1693-9.

19. Meshack AF, Goff DC, Chan W, et al. Comparison of reported symptoms of acute myocardial infarction in Mexican Americans versus non-Hispanic whites (the Corpus Christi Heart Project). Am J Cardiol 1998;82:1329-32.

20. Guarnaccia PJ, Farias P. The social meanings of nervios: A case study of a Central American woman. Soc Sci Med 1988;26:1223-31.

21. Chang L, Toner BB, Fukudo S, et al. Gender, age, society, culture, and the patient's perspective in the functional gastrointestinal disorders. Gastroenterology 2006;130:1435-46.

22. Carrillo JE, Green AR, Betancourt JR. Cross-cultural primary care: A patient-based approach. Ann Intern Med 1999;130:829-34. 
23. American Psychiatric Association. Diagnostics and Statistical Manual of Mental Disorders, Fourth Edition - Text Revision. Washington, DC: American Psychiatric Association, 2000.

24. Population Reference Bureau. 2005 World Population Data Sheet, 2005. <www.prb.org/pdf05/05WorldDataSheet_Eng.pdf> (Version current at April 25, 2007).

25. Licence Services, Marketing Division, Statistics Canada. 2001 Census Handbook. Ottawa: Minister of Industry, 2003.

26. Byrne MM, Souchek J, Richardson M, Suarez-Almazor M. Racial/ethnic differences in preferences for total knee replacement surgery. J Clin Epidemiol 2006;59:1078-86.

27. Zborowski M. Cultural components in response to pain. J Soc Issues 1952;8:16-30.

28. Campbell CM, Edwards RR, Fillingim RB. Ethnic differences in responses to multiple experimental pain stimuli. Pain 2005;113:20-6.

29. Neumann L, Buskila D. Ethnocultural and educational differences in Israeli women correlate with pain perception in fibromyalgia. J Rheumatol 1998;25:1369-73.

30. Anthony M, Berg MJ. Biologic and molecular mechanisms for sex differences in pharmacokinetics, pharmacodynamics, and pharmacogenetics: Part I. J Womens Health Gend Based Med 2002;11:601-15.

31. Jordan JM. Effect of race and ethnicity on outcomes in arthritis and rheumatic conditions. Curr Opin Rheumatol 1999;11:98-103.
32. Green CR. Racial disparities in access to pain treatment. Pain: Clinical Updates 2004;XII:1-4.

33. Rupp T, Delaney KA. Inadequate analgesia in emergency medicine. Ann Emerg Med 2004;43:494-503.

34. Williams DA. Racial and ethnic identifiers in pain management: The importance to research, clinical practice and public health policy. American Pain Society, 2004. < http://www.ampainsoc.org/ advocacy/ethnoracial.htm $>$ (Version current at April 17, 2007).

35. Dworkin SF. Illness behavior and dysfunction: Review of concepts and application to chronic pain. Can J Physiol Pharmacol 1991;69:662-71.

36. Bates MS, Edwards WT, Anderson KO. Ethnocultural influences on variation in chronic pain perception. Pain 1993;52:101-12.

37. Lee MC, Essoka G. Patient's perception of pain: Comparison between Korean-American and Euro-American obstetric patients. J Cult Divers 1998;5:29-37.

38. Bachiocco V, Credico C, Tiengo M. The pain locus of control orientation in a healthy sample of the Italian population: Sociodemographic modulating factors. J Cult Divers 2002;9:55-62.

39. Gordon C. The effect of cancer pain on quality of life in different ethnic groups: A literature review. Nurse Pract Forum 1997;8:5-13. 


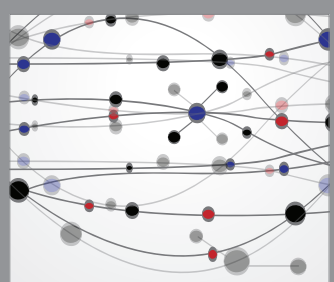

The Scientific World Journal
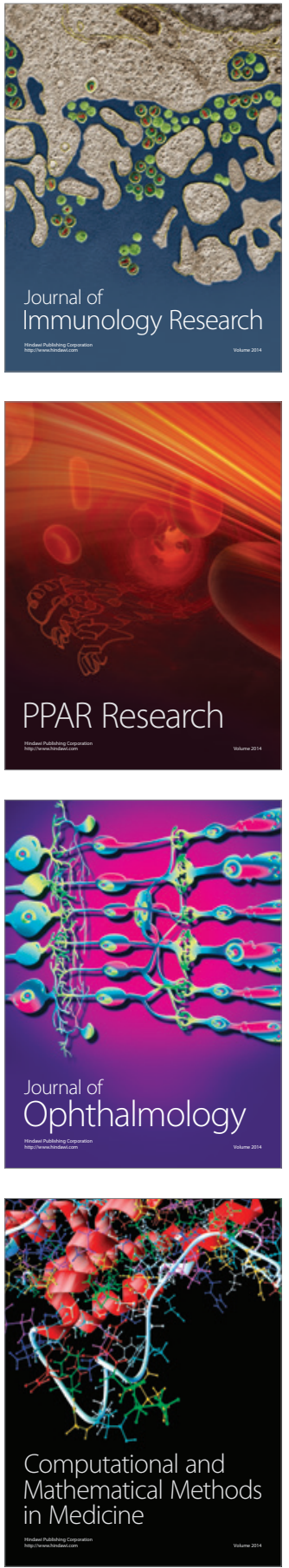

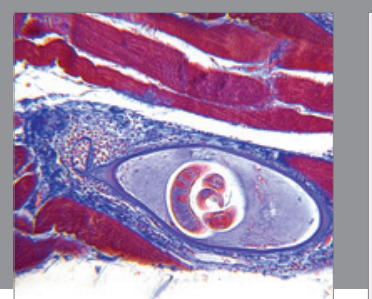

Gastroenterology Research and Practice

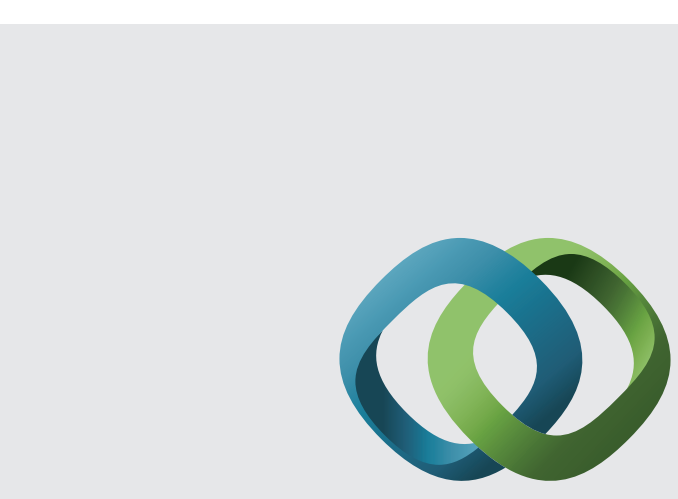

\section{Hindawi}

Submit your manuscripts at

http://www.hindawi.com
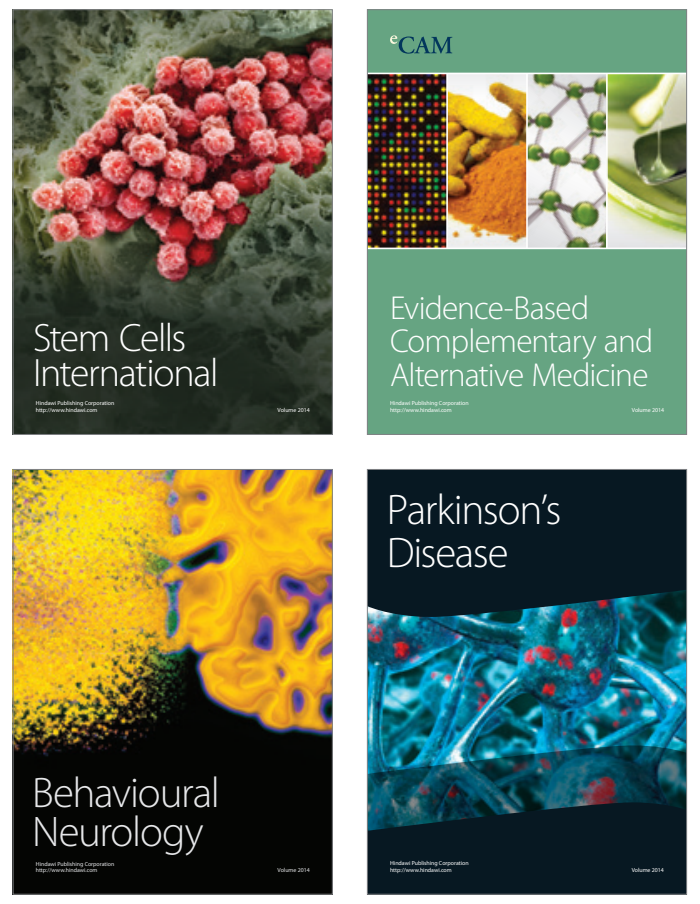
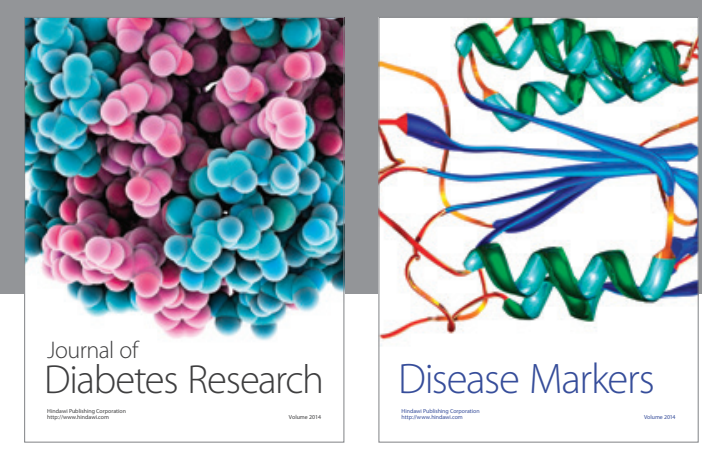

Disease Markers
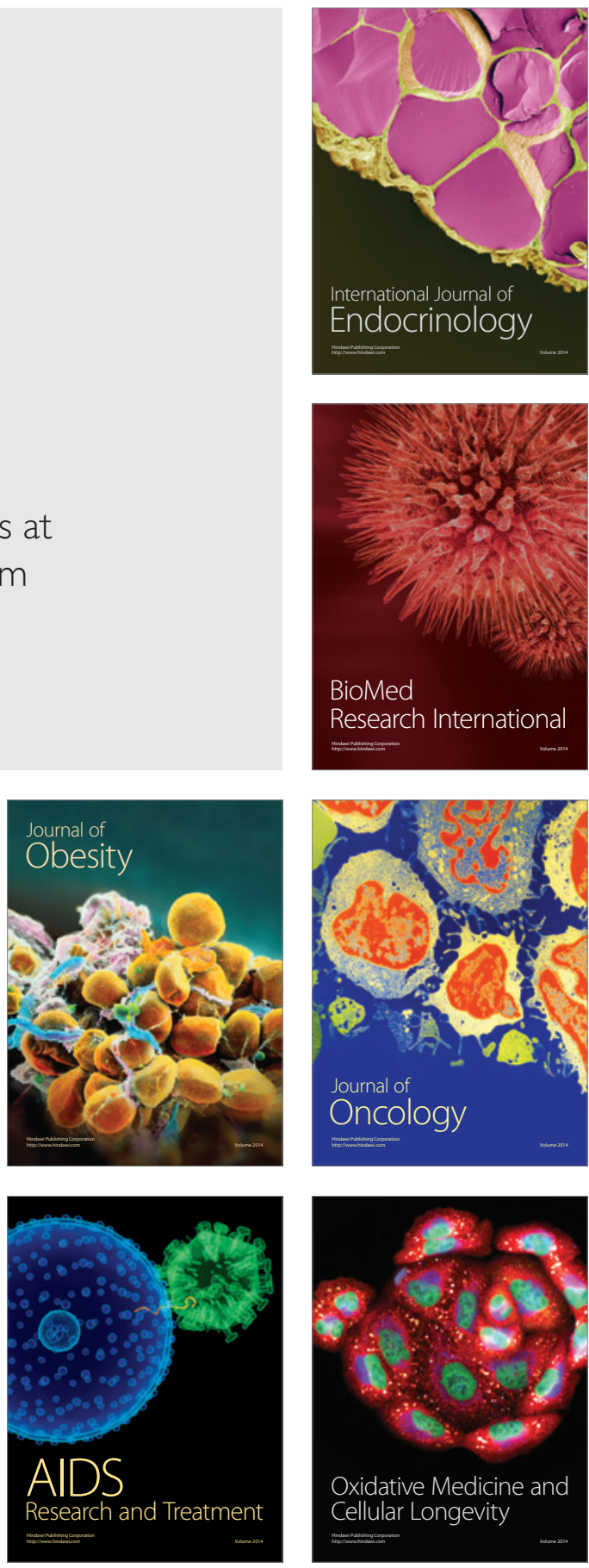